\title{
AN APPROACH FOR PIXEL BASED BINARIZATION OF GRAY IMAGES
}

\author{
Vikash Yadav ${ }^{1}$, Monika Verma ${ }^{2}$, Vandana Dixit Kaushik ${ }^{3}$ \\ ${ }^{1}$ CSE Department, HBTI, Kanpur, India \\ ${ }^{2}$ CSE Department, HBTI, Kanpur, India \\ ${ }^{3}$ CSE Department, HBTI, Kanpur, India
}

\begin{abstract}
This paper presents a hybrid approach for binarization as well as a review on Binarization of images. Binarization is a process by which an image is converted into bits. It is an important step in most document image analysis systems. An image is a set of pixels. Most of the binarization techniques associate a certain intensity value for each and every pixel. A gray image or gray-scale image is just an image which has each pixel of same intensity. That means there is not much difference in color or value information of pixels. Usually, in common terms, a picture in black and white is considered as gray image in which black has least intensity and white have highest.
\end{abstract}

Keywords - Binarization, Images, Segmentation, Restoration, etc

\section{INTRODUCTION}

Over the last 20 years there has been a continued and substantial interest in the field of binarization techniques. In some applications, the use of bi-level images decreases the computational cost of subsequent processing compared to gray-level image information. Binary form is the most common form of data storage [1]. Data in the form of text is extremely rare. Binary files especially images are used for several reasons. Reaction time for binary data is quite less as compared to data in normal character form. Conversion of a larger integer requires some time although the time taken in one conversion is not significant but the accumulated time of multiple. Conversions might result in very slow processing [2]. Applications such as computer games that require large amounts of data to be processed in real time would come to a standstill if there is no binarization of data. Also, the size of a binarized image is much smaller than the actual image, hence lesser requirement of storage space. Also the processing time of smaller image is faster. For example, a DVD would not be able to hold a movie if it were to be stored in its original form, hence it is stored in binary form [3].

\section{LITERATURE REVIEW}

A Comprehensive Review on Analysis of Image Binarization for degraded documents. The research is based on the image binarization of degraded documents. It is challenging to successfully improve the quality of such documents because of the variation in background and foreground text of different document images. The various parameters that are involved when studying the features of these document images are: Stroke width, Stroke Brightness, Stroke Connection and document background [4]. The various steps involved in the binarization of a documented image are Preprocessing, Contrast Image Construction,

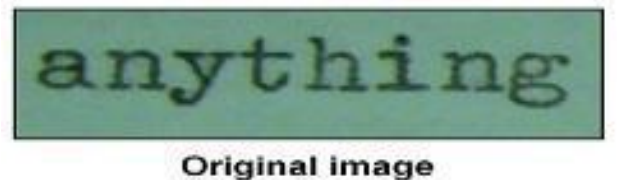

Original image

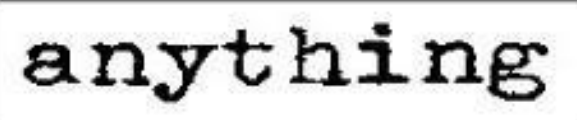

Correct binarization

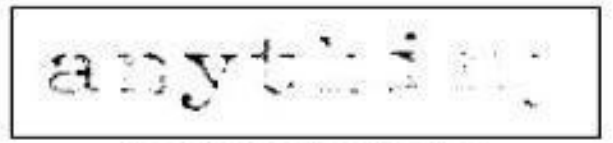

Incorrect binarization

Fig. 1 Example of text image binarization

Edge Detection, Text Stroke Edge Pixel Detection (Ostu's Method/Canny's Edge Detector), Local Threshold Estimation, State of the Art Method. The next step after binarization of an image is its restoration. Restoration is done by degrading the image using prior knowledge of the degraded phenomenon. Once degraded, the inverse is applied to the degraded image in order to extract the original image. Adaptive Degraded Document Image Binarization. Unlike the standard techniques, this is a newer approach to the binarization and improvement of degraded document images [5]. The given method does not utilize any prior parameter tuning by the user and can deal with degraded images which have been degraded either due to shadows, non-uniform illumination, low contrast, or large signaldependant noise or by smear and strain. The steps involved in restoring such images are A preprocessing procedure using a low pass Wiener filter. A rough estimation of foreground regions. A background surface calculation by interpolating neighboring background intensities. A 
threshold calculation by combining the calculated background surface [6]. The above step is done while incorporating image up sampling. A post processing step in order to improve the quality of text regions and preserve stroke connectivity. Robust Document Image Binarization Technique for Degraded Document Images. This study deals with the highly challenging task of segmenting text from badly degraded document images due the very high variation in the background and foreground text of various document images. It is done using a technique known as Adaptive Image Contrast. It is an amalgamation of the local image contrast and local image gradient which in turn is tolerant to text and background variations caused due to the degradations [7]. The steps undertaken in this technique are Construction of Adaptive Contrast map for an input of degraded document image. Now follows the binarization of the contrast map and is then combined with Canny's Edge map in order to identify the text stroke edge pixels. Segmentation of the document text by a local threshold which is estimated based on the intensities of detected text stroke edge pixels within a localized window. The proposed method does not involve any complexities and hence is simple, robust and involves little or no parameter tuning. The given method is also tolerant to uneven illumination and document smear. Best Combination of Binarization Methods for License Plate Character Segmentation. The following study does not focus on any one method for the image segmentation but presents an in-depth analysis of multiple methods that are optimum for the task stated. In a camera based recognition system, there are generally 3 steps that are involved. Sequential process of target region detection Character Segmentation, Character Restoration. The work mainly focuses on the third step which is Character Restoration [8]. Multiple methods involving Character Restoration are used.

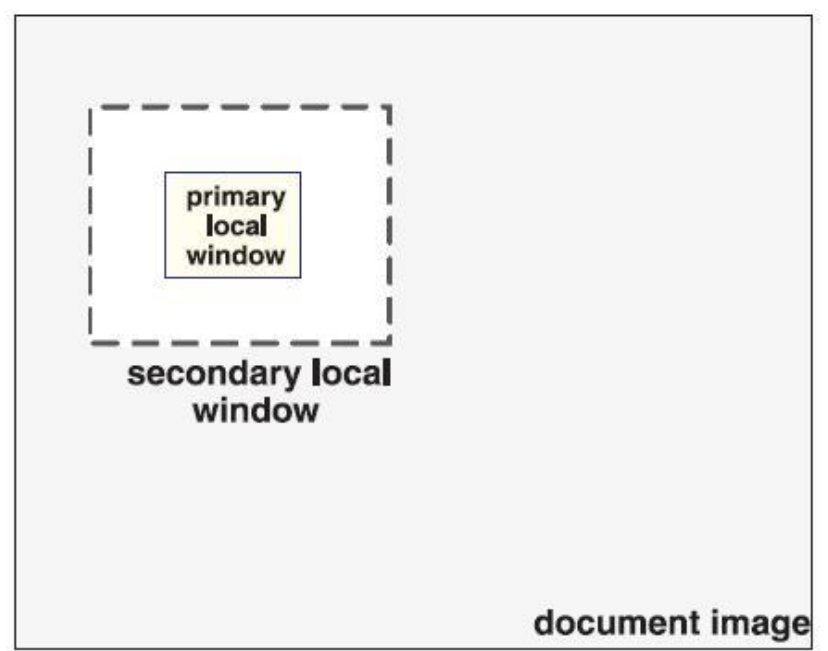

Fig. 2 Primary local window and secondary local window of Feng's technique of binarization

\section{TECHNIQUES FOR BINARIZATION}

We are in process of studying and analyzing some techniques of image binarization, focusing on Feng's Technique, T. R. Singh's Method and Eikvil Technique and we are also trying to design and develop a new technique for better results. There are different parameters on which any binarization technique can be considered effective and optimal. Often, in most cases, parameters of quality, time and efficiency are taken into account. Time taken in process of binarization of an image plays an important role. On the basis of time taken in process, we can classify different technique. The technique which takes least amount of time is considered as an optimal technique [9]. Here, it will be a motive to reduce the time taken in technique to be evolved. The target of this project is to develop an algorithm for binarization of gray scale image which is efficient in quality and also in matter of time. Beside this, to compare sample obtained image after binarization using three mentioned techniques and to find out comparisons. This work primarily includes the study of binarization of gray scale document images done using three approaches or techniques. They are Feng's Technique, T.R. Singh Technique, Eikvil Technique. Feng technique mainly focuses on the binarization of gray scale document images, taking input from mainly PDA's, mobile phones, faxing machines, auto notes taking and OCRs. The proposed method overcomes general problems of poor quality document images, suck as non uniform illumination, undesirable shadows and random noise. T.R. Singh proposed a method of binarizing images by a method called automatic binarization which involves transforming a gray scale image into a binary image without using any standard deviation which is used in Sauvola's technique and Niblack's technique but by adapting to the pixels within a local region environment [10]. It uses local mean. In Eikvil technique, page is considered as a collection of subcomponents such as text, background and picture. The problems caused by noise, illumination and many source type-related degradations are addressed. Two new algorithms are applied to determine a local threshold for each pixel. The performance evaluation of the algorithm utilizes test images with ground-truth, evaluation metrics for binarization of textual and synthetic images, and a weightbased ranking procedure for the final result presentation.

\section{METHODOLOGY FOR BINARIZATION}

Binarization, which scans gray-scale text images into two levels, is usually the first stage in document image understanding systems [11]. This is because the use of bilevel information greatly reduces the computational load and the analysis algorithm complexity. Moreover, binarization is also the most critical stage, since any error in this phase will pass down to the following ones. One of the popular binarization approaches is gray value thresholding and the corresponding techniques can be further classified into global and local thresholding. Each and every pixel of the concerned gray scale input image should be compared with the threshold value and according to it, pixels are separated into two classes' background and foreground. Thus threshold plays a major role in binarization and choosing of an appropriate threshold value is an important task [12].

In Pre-processing stage, there are several sub-steps. First of all steps, which comes in Pre-processing step is selecting original image to be tested. That image is then digitally 
acquired in second step of pre-processing stage, i.e. Image acquisition step. Third step is Image optimization. This step of Pre-processing is further divided into several small processes such as Gray scale conversion, Contrast optimization, Smoothing, Refining edges, etc [14]. After Image optimization, next step is Histogram equalization. In this step, image is optimized by using histogram feature. Fifth step is obtaining Sample. After this step, Processing stage start. The process is shown in figure 6.

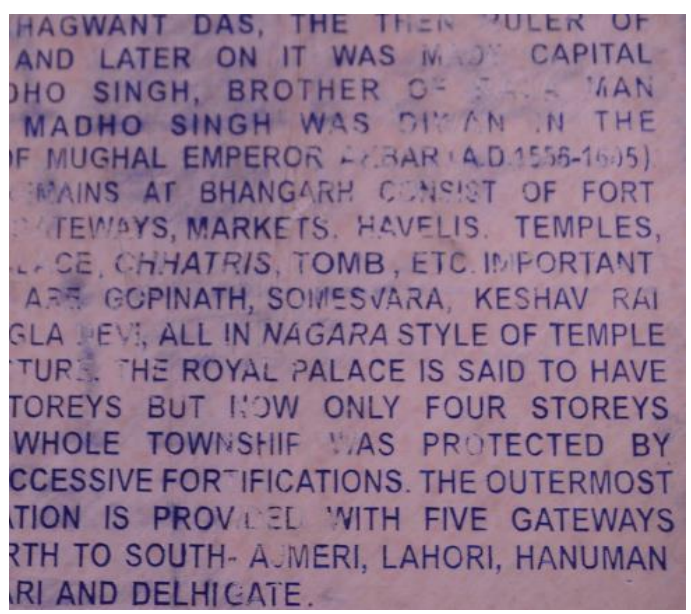

Fig.3 Original Images

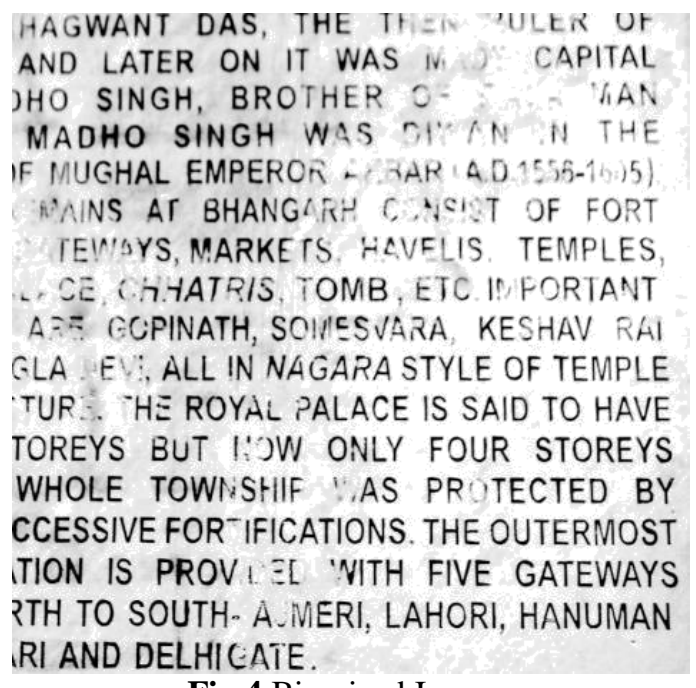

Fig.4 Binarized Images

In this paper we focus on binarizing grayscale documents with the help of local thresholding technique, generally colour documents do not lose much information when converted to grayscale images in context with the differentiation between page foreground and background. Severely degraded or noisy images can be improved drastically using these techniques but unfortunately is slow due to the computational complexity of local mean, max and min from the local region environment of each pixel in the image[13]. The difference between original image and binarized image can me seen in figure 3 and figure 4 respectively. The project is divided into basically three steps. Basically, here, whole process is categorized into three major parts. They are Pre-processing, Processing and Binarization which can be seen in figure 5.

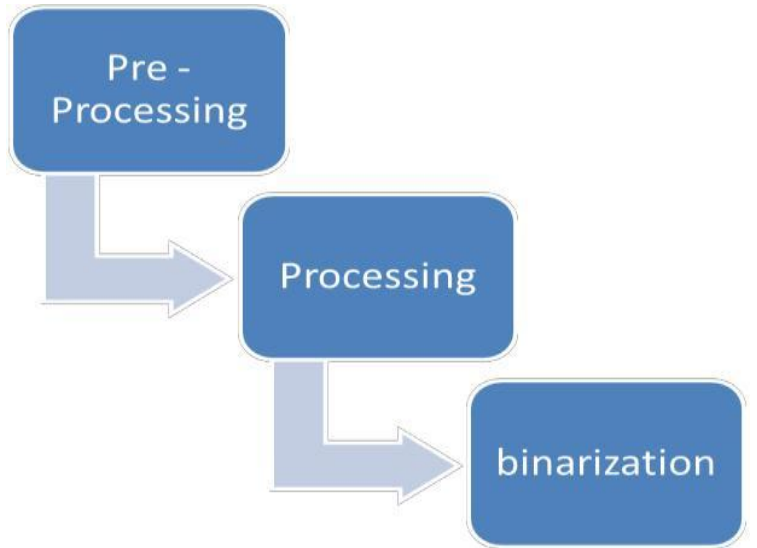

Fig.5 Steps of overall process.

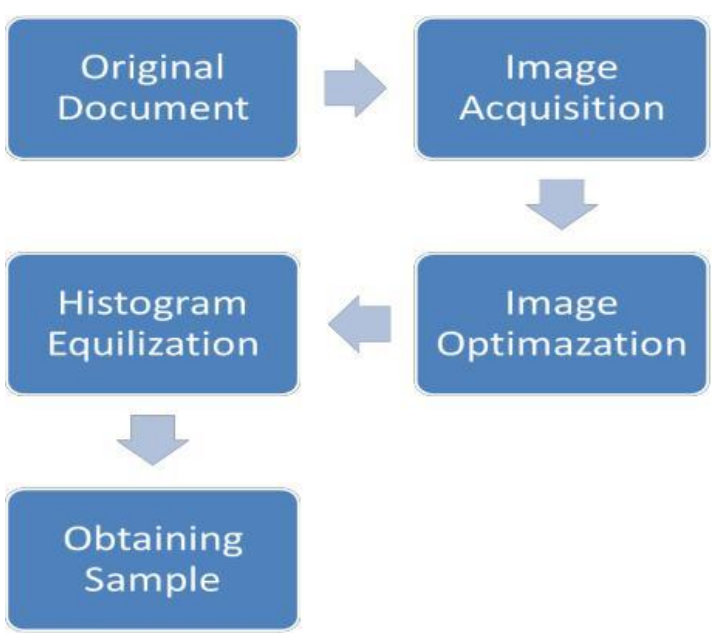

Fig.6 Steps involved in Pre-Processing stage

\section{TOOLS REQUIRED}

Matlab is a very important tool in image processing. The basic data structure in MATLAB is the array, an ordered set of real or complex elements. This object is naturally suited to the representation of images, real-valued, ordered sets of color or intensity data. Java Development Kit (JDK)Image processing stands with one foot firmly in mathematics and the other in aesthetics, and is a critical component of graphical computer systems. Friendly platform such as Java is helpful in image processing. Photoshop an interactive and easy to use application which is very popular for image editing [15].

\section{CONCLUSION \& FUTURE SCOPE}

Binarization of images is a very progressive field in day today and future to come. There is lot of improvements going on in field of binarization. Future is waiting for multi dimensional imaging media. For that, it is required to convert images and document images in binarized form. Documents and images which have non uniform brightness, needs local threshold on small level. The local threshold varies according to various image conditions. 


\section{REFERENCES}

[1] Meng-Ling Feng and Yap-Peng Tan, "Contrast Adaptive Binarization of Low Quality Document Images" The Institute of Electronics, Information and Communication Engineers (IEICE) Electronic express, Volume 1, Issue No. 16, November 2004, Page 501-506.

[2] Chien-Hsing Chou, Wen-HsiungLin and FuChang, "A binarization method with learning-built rules for document images produced by cameras", Pattern Recognition 43, 2010, 1518-1530.

[3] P. Subashini and N. Sridevi, "An Optimal Binarization Algorithm Based on Particle Swarm Optimization", International Journal of Soft Computing and Engineering (IJSCE), Volume-1, Issue-4, September 2011.

[4] S.S. Bedi and Rati Khandelwal, "International Journal of Advanced Research in Computer and Communication Engineering”, International Journal of Soft Computing and Engineering (IJSCE), Vol. 2, Issue 3, March 2013..

[5] O. Imocha Singh, O. James, Tejmani Sinam and T.Romen Singh, "Local Contrast and Mean based Thresholding Technique in Image Binarization", International Journal of Computer Applications, Volume 51- No.6, August 2012.

[6] Rajesh K. Bawa and Ganesh K. Sethi, "A Binarization Technique For Extraction Of Devanagari Text From Camera Based Images", Signal \& Image Processing : An International Journal (SIPIJ), Vol.5, No.2, April 2014.

[7] Youngwoo Yoon, Kyu-Dae Ban, Hosub Yoon, Jaeyeon Lee and Jaehong Kim, "Best Combination of Binarization Methods for License Plate Character Segmentation", Electronics and Telecommunications Research Institute (ETRI) Journal, Volume 35, Number 3, June 2013..

[8] Ntogas, Nikolaos, Ventzas, Dimitrios, "A Binarization Algorithm For Historical Manuscripts", 12th WSEAS International Conference on COMMUNICATIONS, Heraklion, Greece, July 23$25,2008$.

[9] Geetanjali Thakur, “A Comprehensive Review On Analysis Of Image Binarization For Degraded Documents", International Journal of Advance Research In Science And Engineering (IJARSE), Vol. No.3, Issue No.7, July 2014 ISSN-2319-8354(E), Page 325.

[10] Bolan Su, Shijian Lu, and Chew Lim Tan, "Robust Document Image Binarization Technique for Degraded Document Images", IEEE Transactions On Image Processing, Vol. 22, No. 4, April 2013.

[11] Aroop Mukherjee and Soumen Kanrar, "Enhancement of Image Resolution by Binarization", International Journal of Computer Applications, Volume 10- No.10, November 2010.

[12] B. Gatos, I. Pratikakis and S.J. Perantonis, "Adaptive degraded document image binarization", Computational Intelligence. Vol. 4, Issue 2, December 2009.
[13] Chirag Patel, Dr. Atul Patel and Dr. Dipti Shah, "Threshold Based Image Binarization Technique for Number Plate Segmentation", International Journal of Advanced Research in Computer Science and Software Engineering, Volume 3, Issue 7, July 2013, Page 108-114.

[14] T. Romen Singh, Sudipta Roy and Kh. Manglem Singh, "Histogram Domain Adaptive Power Law Applications in Image Enhancement Technique", International Journal of Computer Science and Information Technologies (IJCSIT), Vol. 5, Issue 3, 20140.

[15] Rukhsar Firdousi and Shaheen Parveen, "Local Thresholding Techniques in Image Binarization" International Journal Of Engineering And Computer Science, Volume 3, Issue 3, March 2014, Page No. 4062-4065. 